\title{
ORFEUS Observation of the Central Star of NGC 6543
}

J. Zweigle ${ }^{1}$ ，M. Grewing ${ }^{1,2}$ ，J. Barnstedt ${ }^{2}$ ，M. Gölz ${ }^{2}$ ，W. Gringel ${ }^{2}$ ，C. Haas ${ }^{2}$, W. Hopfensitz ${ }^{2}$, N. Kappelmann ${ }^{2}$, G. Krämer ${ }^{2}$, I. Appenzeller ${ }^{3}$, J. Krautter ${ }^{3}$ and H. Mandel ${ }^{3}$

${ }^{1}$ Institut de Radio Astronomie Millimétrique (IRAM);

${ }^{2}$ Institut für Astronomie und Astrophysik der Universität Tübingen, Abt. Astronomie;

${ }^{3}$ Landessternwarte Heidelberg-Königstuhl

During the ORFEUS-SPAS (Orbiting Retrievable Far and Extreme Ultraviolet Spectrometer on the Shuttle Pallet Satellite) mission STS-51, flown in September 1993, we observed the central star of the planetary nebula NGC 6543 in the far ultraviolet $(90 \mathrm{~nm}$ to $115 \mathrm{~nm})$ wavelength region using the University of California, Berkeley spectrometer with a spectral resolution of $0.03 \mathrm{~nm}$.

In addition to narrow absorption lines of atomic and molecular species (e.g. H I, C I, NI, OI, $\mathrm{H}_{2}$ ) the spectrum shows strong P-Cygni-profiles of the S VI $(93.3 \mathrm{~nm}, 94.4 \mathrm{~nm})$, OVI $(103.2 \mathrm{~nm}, 103.8 \mathrm{~nm})$ and PV $(111.8 \mathrm{~nm}, 112.8 \mathrm{~nm})$ resonance doublets. The terminal wind velocities for the different ions are $1550 \mathrm{~km} / \mathrm{s}$ for S VI, $1970 \mathrm{~km} / \mathrm{s}$ for O VI and $1550 \mathrm{~km} / \mathrm{s}$ for PV. The analysis of these three P-Cygni line doublets using the escape probability method yields as a mean value for the lower limit of the central star's mass loss rate $1.0 \cdot 10^{-8} M_{\odot} /$ yr.

\section{Acknowledgement}

The ORFEUS-SPAS mission could not have succeeded without the dedicated work of the hardware groups at Tübingen, Heidelberg and Berkeley and the effort and efficient support by NASA, DARA, DASA and Kayser-Threde. This research was supported by the BMFT/BMBF (DARA) through grant 01 OS 8501 and NASA grant NAG 5-696. 